\title{
Myocarditis-A Quick Review
}

\author{
Dr. Umesh Prasad ${ }^{1}$, Dr. Nishu Kumar ${ }^{2}$ \\ ${ }^{I}$ Department Of Medicine/ Associate Professor/ Rims/Ranchi University/India \\ ${ }^{2}$ Department Of Medicine/ Junior Resident/ Rims/Ranchi University/India
}

\section{Myocarditis(Inflammation Of The Heart)}

It can result from multiple causes but most commonly attributed to infective agents that can injure the myocardium through direct invasion, production of cardiotoxic substances, or chronic inflammation with or without persistent infection. Myocarditis may present with a wide range of symptoms, ranging from mild dyspnea or chest pain that resolves without specific therapy to cardiogenic shock and death. Dilated cardiomyopathy with chronic heart failure is the major long-term sequela of myocarditis. Most often, myocarditis results from common viral infections; less commonly, specific forms of myocarditis may result from other pathogens, toxic or hypersensitivity drug reactions, giant-cell myocarditis, or sarcoidosis. The prognosis and treatment of myocarditis vary according to the cause, and clinical and hemodynamic data usually provide guidance to decide when to refer a patient to a specialist for endomyocardial biopsy. The aim of this review is to provide a practical and current approach to the evaluation and treatment of suspected myocarditis.

\section{Definition}

The standard Dallas pathological criteria for the definition of myocarditis require that an inflammatory cellular infiltrate with or without associated myocyte necrosis be present on conventionally stained heart-tissue sections ${ }^{1}$. These criteria are limited by variability in interpretation, lack of prognostic value, and low sensitivity, in part due to sampling error ${ }^{2,3}$. These limitations have led to alternative pathological classifications with criteria that rely on cell-specific immunoperoxidase stains for surface antigens, such as anti-CD3, anti-CD4, anti-CD20, anti-CD68, and anti-human leukocyte antigen ${ }^{4,5}$. Criteria that are based on immunoperoxidase staining have greater sensitivity and may have prognostic value. ${ }^{6}$ Preliminary studies suggest that noninvasive cardiac magnetic resonance imaging (MRI) may provide an alternative method for diagnosis without the risks of biopsy. For example, regions of myocarditis are reported to correlate closely with regions of abnormal signal on cardiac MRI. ${ }^{7,8}$ The lack of consensus regarding the value of invasive studies such as endomyocardial biopsy and the overall good prognosis for patients with mild, acute dilated cardiomyopathy who have suspected myocarditis have led to recent recommendations that endomyocardial biopsy should be considered on the basis of the likelihood of finding specific treatable disorders. ${ }^{9}$

\section{Clinical Features And Incidence}

Acute myocarditis is frequently first diagnosed as nonischemic dilated cardiomyopathy in a patient with symptoms that have been present for a few weeks to several months. However, manifestations range from subclinical disease to sudden death, with new-onset atrial or ventricular arrhythmias, complete heart block, or an acute myocardial infarction-like syndrome. Cardiac symptoms are variable and may include fatigue, decreased exercise tolerance, palpitations, precordial chest pain, and syncope. Chest pain in acute myocarditis can result from an associated pericarditis or, occasionally, from coronary-artery spasm. ${ }^{10}$

The true incidence of myocarditis in the community is unknown. Endomyocardial biopsy is used infrequently because of perceived risks and the lack of a widely accepted and sensitive histologic standard. Seroepidemiologic data are difficult to interpret because of the heterotopic effect of enteroviruses, which may cause an amnestic antibody response to other coxsackievirus B strains.However, the observation that viral genomes are more common in cardiac tissue from patients with chronic dilated cardiomyopathy than in tissue from patients with valvular or ischemic cardiomyopathy supports the concept that viral myocarditis leads to a substantial disease burden in the community. Furthermore, myocarditis is an important cause of sudden death, as well as childhood cardiomyopathy. 


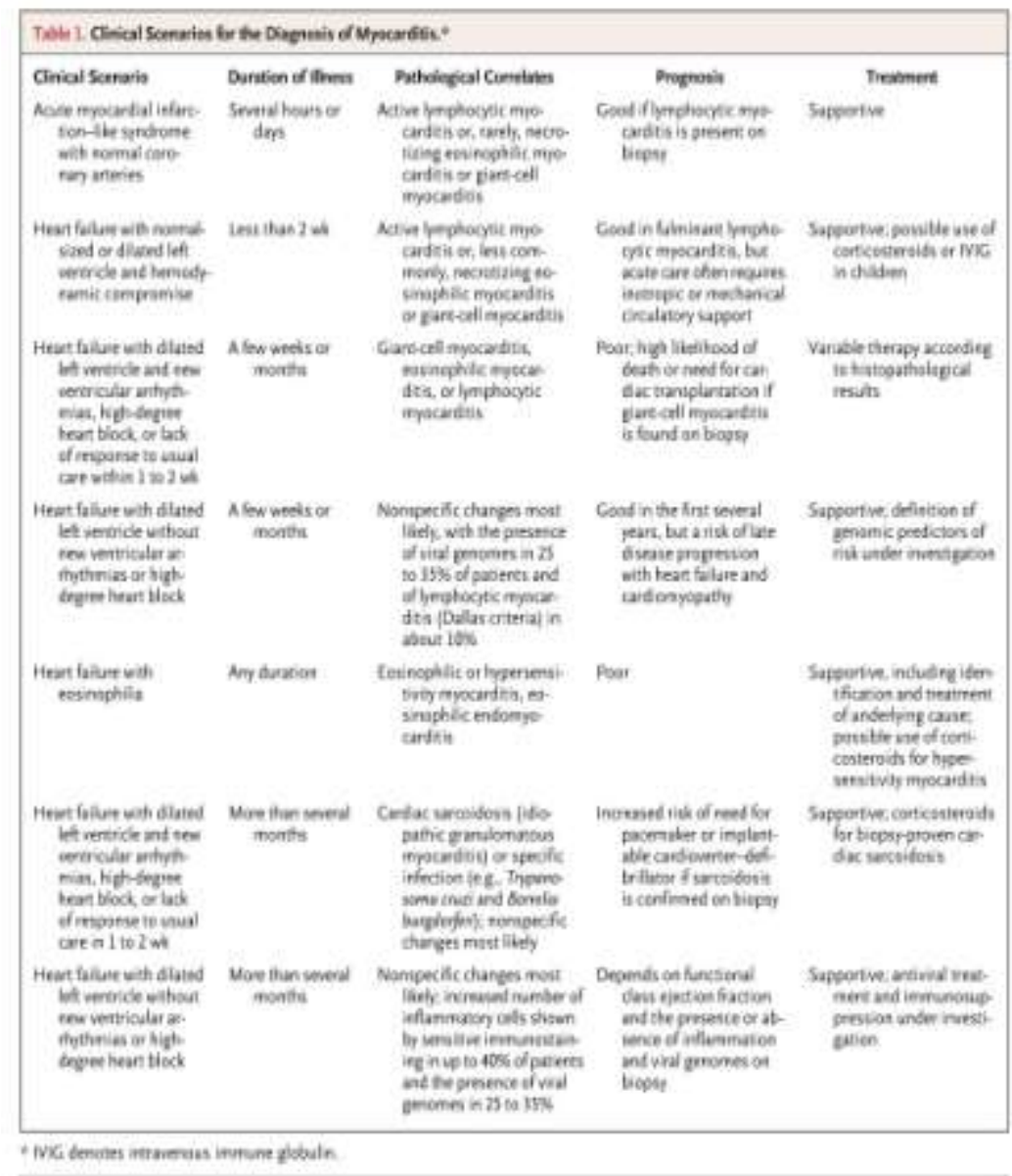

\section{Etiology}

Viral and postviral myocarditis remain major causes of acute and chronic dilated cardiomyopathy. Seroepidemiologic and molecular studies linked coxsackievirus B to outbreaks of myocarditis from the 1950s through the 1990s. The spectrum of viruses that were detected in endomyocardial biopsy samples shifted from coxsackievirus B to adenovirus in the late 1990s and, in the past 5 years, to parvovirus B19 and other viruses, according to reports from the United States and Germany ${ }^{11}$. Many other viruses have also been associated less frequently with myocarditis; these viruses include Epstein-Barr virus, cytomegalovirus, and human herpesvirus 6. Myocarditis can result from infection with Borrelia burgdorferi (Lyme disease).

Myocarditis is the most common cardiac pathological finding at autopsy of patients infected with the human immunodeficiency virus (HIV), with a prevalence of $50 \%$ or more ${ }^{12}$. Drug-induced hypersensitivity reactions and systemic hypereosinophilic syndromes can cause a specific myocarditis . Numerous medications, including some anticonvulsants, antibiotics, and antipsychotics, have been implicated in hypersensitivity myocarditis. Eosinophilic myocarditis is characterized by a predominantly eosinophilic infiltrate in the myocardium and may occur in association with systemic diseases, such as the hypereosinophilic syndrome, the Churg-Strauss syndrome, Löffler's endomyocardial fibrosis, cancer, and parasitic, helminthic, or protozoal infections $^{13,14,15}$. Two idiopathic and histologically similar disorders, giant-cell myocarditis and cardiac sarcoidosis, are rare but important causes of cardiomyopathy.

\section{Diagnosis}

Biomarkers of cardiac injury are elevated in a minority of patients with acute myocarditis but may help confirm the diagnosis. Troponin I has high specificity (89\%) but limited sensitivity (34\%) in the diagnosis of myocarditis ${ }^{16}$. Clinical and experimental data suggest that increased levels of cardiac troponin I are more common than increased levels of creatine kinase $\mathrm{MB}$ in acute myocarditis. ${ }^{17} \mathrm{~A}$ few serologic and imaging biomarkers have been associated with poor clinical outcome. For example, relatively high serum levels of Fas ligand and interleukin-10 may predict an increased risk of death ${ }^{18,19}$ although these assays are not widely available. 
In acute myocarditis, the electrocardiogram may show sinus tachycardia with nonspecific ST-segment and T-wave abnormalities. Occasionally, the changes on electrocardiography are suggestive of an acute myocardial infarction and may include ST-segment elevation, ST-segment depression, and pathologic Q waves. Pericarditis not infrequently accompanies myocarditis clinically and is often manifested in pericarditis-like changes seen on electrocardiography. The sensitivity of the electrocardiogram for myocarditis is low $(47 \%) .{ }^{20}$ The presence of Q waves or left bundle-branch block is associated with higher rates of death or cardiac transplantation. $^{21}$

Echocardiography is useful primarily to rule out other causes of heart failure, since there are no specific features of acute myocarditis. Echocardiographic patterns of dilated, hypertrophic, restrictive, and ischemic cardiomyopathies have been described in histologically proven myocarditis. Segmental or global wall-motion abnormalities in myocarditis can simulate myocardial infarction. ${ }^{22}$ In the Myocarditis Treatment Trial, increased sphericity and left ventricular volume occurred in acute, active myocarditis. ${ }^{23}$ Fulminant myocarditis may be distinguished from acute myocarditis by a smaller left ventricular cavity size and increased wall thickness. ${ }^{24}$ The loss of right ventricular function was the most powerful predictor of death or the need for cardiac transplantation in a series of 25 patients with biopsy-confirmed myocarditis. ${ }^{25}$

Cardiac MRI is being used with increasing frequency as a diagnostic test in suspected acute myocarditis ${ }^{26,27}$ and may be used to localize sites for endomyocardial biopsy Endomyocardial biopsy should be performed in patients with unexplained, new-onset heart failure of less than 2 weeks' duration in association with a normal-size or dilated left ventricle and hemodynamic compromise, for suspected fulminant myocarditis. Endomyocardial biopsy should also be performed in patients with unexplained, new-onset heart failure of 2 weeks' to 3 months' duration in association with a dilated left ventricle and new ventricular arrhythmias or Mobitz type II or second-degree or third-degree heart block and in patients who do not have a response to usual care within 1 to 2 weeks, for suspected giant-cell myocarditis.
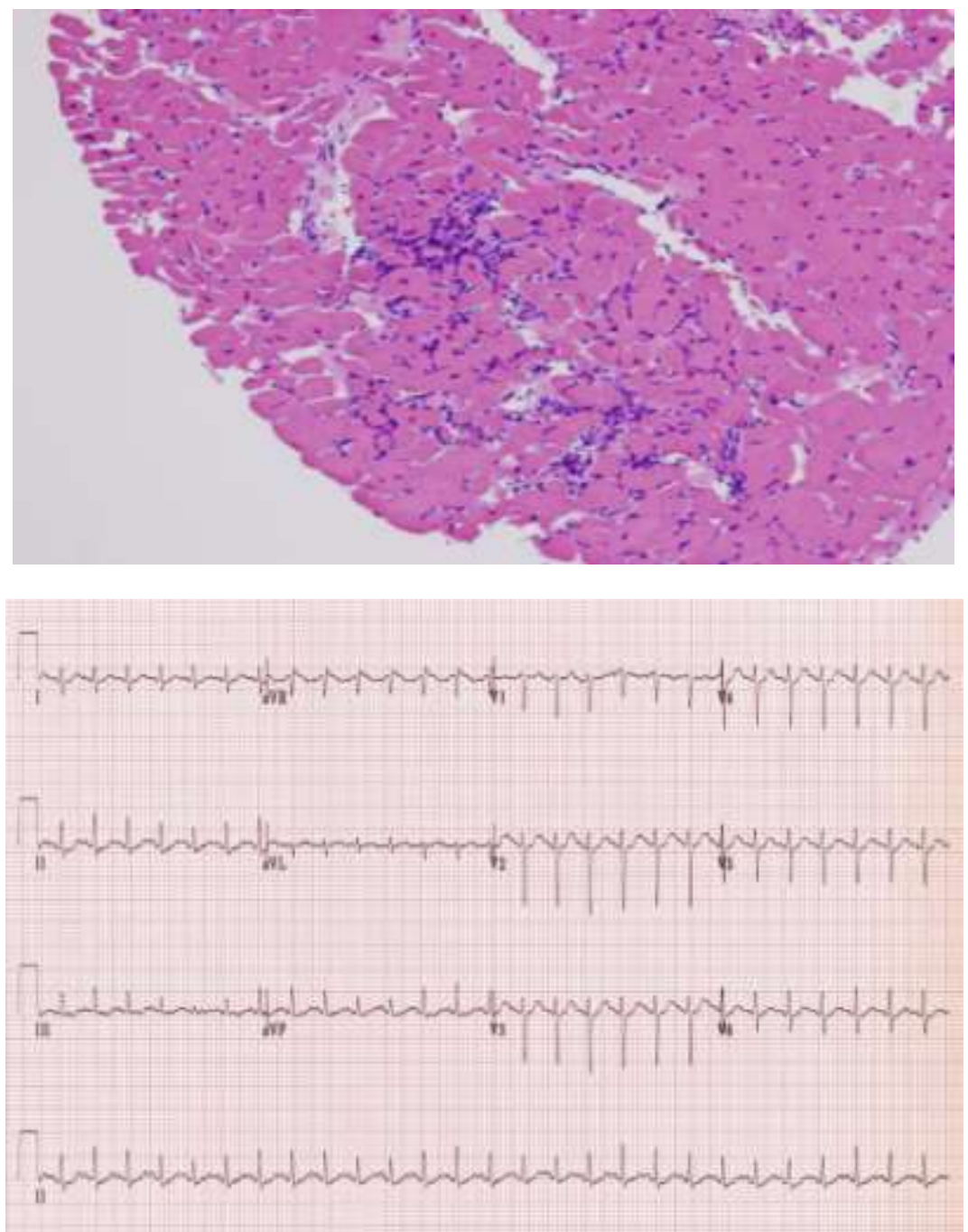

Fig 1:H and E, low power, showing numerous lymphocytes with associated myocyte damage in myocarditis 
Fig 2: Sinus tachycardia with non-specific ST segment changes seen in acute myocarditis.
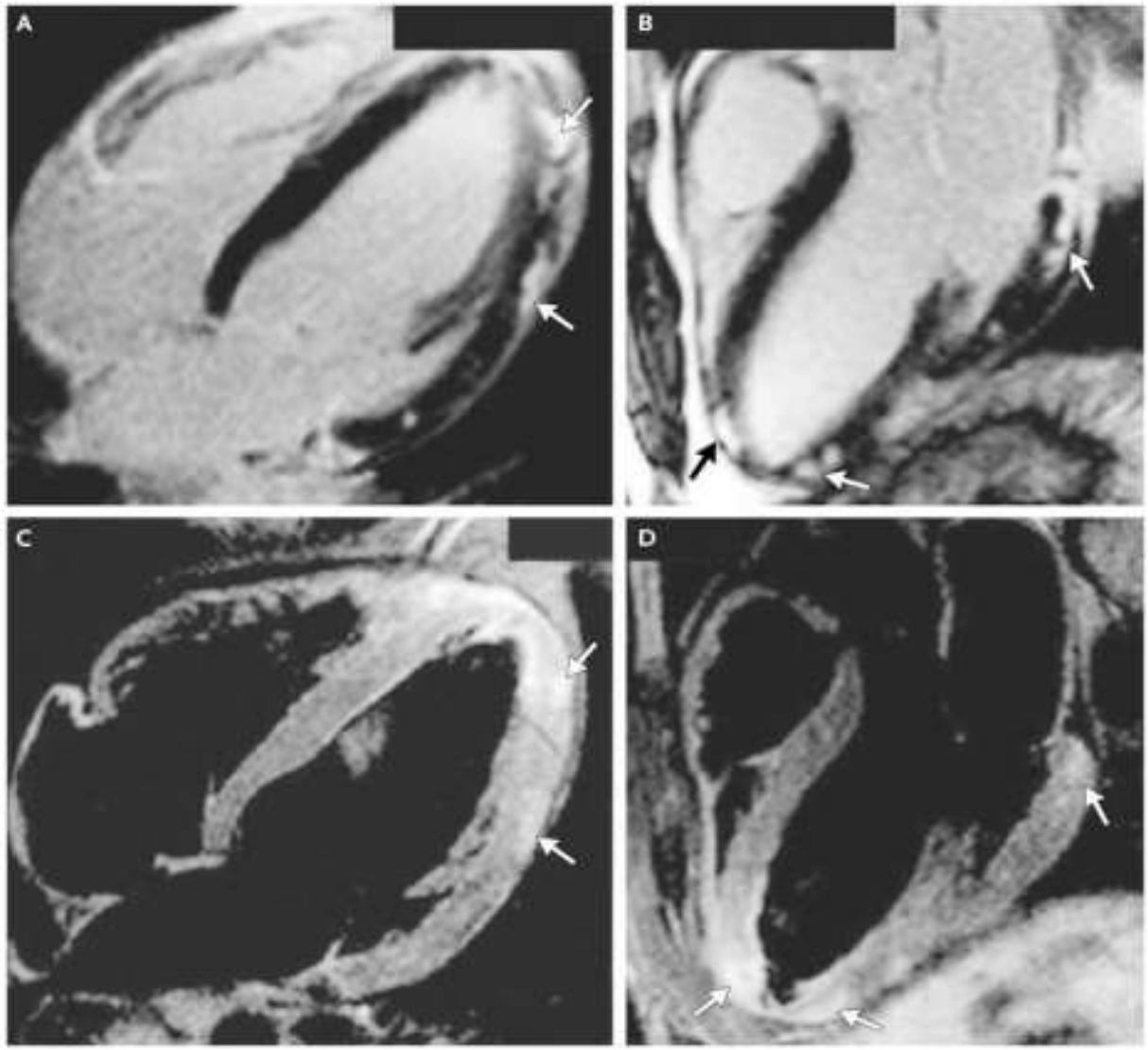

Fig 3: Contrast-Enhanced Magnetic Resonance Imaging (MRI) of the Heart of a 24-Year-Old Man with Acute Myocarditis.Cardiac MRI is being increasingly used to evaluate suspected acute myocarditis and to localize sites for endomyocardial biopsy, with additional detail shown with delayed gadolinium enhancement (Panel A, arrows), in a four-chamber view (Panel B, arrows), and in T2-weighted three-chamber views (Panels C and D, arrows).

\section{Treatment}

The mainstay of therapy for acute myocarditis is supportive therapy for left ventricular dysfunction. Most patients will improve with a standard heart-failure regimen that includes the administration of angiotensin-converting-enzyme inhibitors or angiotensin-receptor blockers, beta-blockers such as metoprolol and carvedilol, and diuretics, if needed. In patients whose condition deteriorates despite optimal medical management, case series suggest a role for mechanical circulatory support, such as ventricular assist devices or extracorporeal membrane oxygenation, as a bridge to transplantation or recovery.

Since no clinical trials of therapy for heart failure have been conducted specifically in patients with myocarditis, the only relevant studies describe animal models. Patients recovering from acute myocarditis should refrain from aerobic activity for a period of months after the clinical onset of the disease, based on studies in rodents with myocarditis in which increased death rates were associated with sustained exercise. ${ }^{28}$ The reintroduction of aerobic activities somewhat depends on the severity of left ventricular dysfunction and the extent of recovery. ${ }^{29}$ The use of candesartan improved survival in a murine model of viral myocarditis $(60 \%$, vs. $18 \%$ with no candesartan treatment). ${ }^{30}$ The use of carteolol, a nonselective beta-blocker, improved histopathological results and reduced wall thickness in coxsackievirus B myocarditis. ${ }^{31}$ The use of nonsteroidalantiinflammatory drugs was associated with increased mortality. ${ }^{32,33,34}$ Taken together, these data support the application of the current heart-failure guidelines to patients with heart failure from myocarditis.

In patients with acute myocarditis, therapy for arrhythmias is also supportive, since such arrhythmias usually resolve after the acute phase of the disease, which can last several weeks. However, in acute myocarditis, temporary pacemakers may be required for patients with symptomatic bradycardia or complete heart block. Patients with symptomatic or sustained ventricular arrhythmias may need amiodarone and possibly an implantable cardioverter-defibrillator, even if active inflammation is still present. The prognostic importance and treatment of nonsustained ventricular arrhythmias in acute myocarditis have not been systematically evaluated. 
Antiviral and immunomodulatory effects that have been shown in experimental models and uncontrolled case series suggest that intravenous immune globulin (IVIG) might have a therapeutic use in myocarditis. However, in the Intervention in Myocarditis and Acute Cardiomyopathy trial, patients with acute dilated cardiomyopathy who were treated with IVIG did no better than those given placebo. ${ }^{35}$ Therefore, the routine use of IVIG for acute myocarditis in adults is not recommended. IVIG has not been evaluated rigorously for the treatment of chronic dilated cardiomyopathy with inflammation or viral persistence. IVIG may have a role in the treatment of acute pediatric myocarditis. ${ }^{36}$

Results from several randomized, controlled trials of immunosuppression for acute myocarditis were negative or only marginally positive. ${ }^{37}$ These studies suggest that immunosuppression is not beneficial in the routine treatment of acute lymphocytic myocarditis. Future trials involving patients with acute myocarditis are probably not feasible since the disease affects so few patients, has a highly variable clinical prognosis, and is associated with substantial improvement in left ventricular function with usual care. ${ }^{38}$ Unlike lymphocytic myocarditis, transplant-free survival in patients with giant-cell myocarditis may be prolonged with a combination of cyclosporine and corticosteroids. ${ }^{39}$ There may be a broader role for immunosuppression in patients with chronic, moderate-to-severe cardiomyopathy, whose condition is unlikely to improve further after optimal care has been given for 6 to 12 months. In one trial involving 84 patients with chronic dilated cardiomyopathy and human leukocyte antigen expression on cardiomyocytes, the use of azathioprine and prednisone was associated with improvement in cardiac function and in New York Heart Association functional class. ${ }^{40}$ Other approaches to modify immune activation that are under investigation in this population include immunoadsorption and immunomodulation.

\section{Summary And Future Directions}

This review discusses an approach to suspected myocarditis according to the likelihood of finding a treatable disorder. A major issue for the future is whether the diagnosis of myocarditis will continue to require histologic confirmation. Cardiac MRI is a promising tool but requires additional validation for noninvasive diagnosis and prognosis in acute and chronic myocarditis. On the horizon, analysis of messenger RNA and protein markers from peripheral-blood components may be able to detect a clinically meaningful inflammatory signal in high-risk populations without the risk of endomyocardial biopsy. ${ }^{41}$ Treatment of subpopulations of chronic viral-associated and nonviral myocarditis with biopsy-guided therapy is an area of active investigation. Our understanding of the immunologic regulation of viral cardiac infection is derived primarily from research in animal models. The insights from these models may be explored in human studies in the next decade to develop new diagnostic tests and possibly pathway-specific treatments.

\section{References}

[1]. Aretz HT, Billingham ME, Edwards WD, et al. Myocarditis: a histopathologic definition and classification. Am J CardiovascPathol 1987;1:3-14

[2]. Chow LH, Radio SJ, Sears TD, McManus BM. Insensitivity of right ventricular endomyocardial biopsy in the diagnosis of myocarditis. J Am CollCardiol 1989;14:915-920

[3]. Baughman KL. Diagnosis of myocarditis: death of Dallas criteria. Circulation 2006;113:593-595

[4]. Herskowitz A, Ahmed-Ansari A, Neumann DA, et al. Induction of major histocompatibility complex antigens within the myocardium of patients with active myocarditis: a nonhistologic marker of myocarditis. J Am CollCardiol 1990;15:624-632

[5]. Maisch B, Portig I, Ristic A, Hufnagel G, Pankuweit S. Definition of inflammatory cardiomyopathy (myocarditis): on the way to consensus: a status report. Herz 2000;25:200-209

[6]. Kindermann I, Kindermann M, Kandolf R, et al. Predictors of outcome in patients with suspected myocarditis. Circulation 2008;118:639-648[Erratum, Circulation 2008;118(12):e493.]

[7]. Mahrholdt H, Goedecke C, Wagner A, et al. Cardiovascular magnetic resonance assessment of human myocarditis: a comparison to histology and molecular pathology. Circulation 2004;109:1250-1258

[8]. Gutberlet M, Spors B, Thoma T, et al. Suspected chronic myocarditis at cardiac MR: diagnostic accuracy and association with immunohistologically detected inflammation and viral persistence. Radiology 2008;246:401-409

[9]. Cooper LT, Baughman KL, Feldman AM, et al. The role of endomyocardial biopsy in the management of cardiovascular disease: a scientific statement from the American Heart Association, the American College of Cardiology, and the European Society of Cardiology. Circulation 2007;116:2216-2233

[10]. McCully RB, Cooper LT, Schreiter S. Coronary artery spasm in lymphocytic myocarditis: a rare cause of acute myocardial infarction. Heart 2005;91:202-202

[11]. Kuhl U, Pauschinger M, Noutsias M, et al. High prevalence of viral genomes and multiple viral infections in the myocardium of adults with "idiopathic" left ventricular dysfunction. Circulation 2005;111:887-893

[12]. Taliercio CP, Olney BA, Lie JT. Myocarditis related to drug hypersensitivity. Mayo ClinProc 1985;60:463-468

[13]. Corssmit EP, Trip MD, Durrer JD. Löffler'sendomyocarditis in the idiopathic hypereosinophilic syndrome. Cardiology 1999;91:272-276

[14]. Spodick DH. Eosinophilic myocarditis. Mayo ClinProc 1997;72:996-996

[15]. Corradi D, Vaglio A, Maestri R, et al. Eosinophilic myocarditis in a patient with idiopathic hypereosinophilic syndrome: insights into mechanisms of myocardial cell death. Hum Pathol 2004;35:1160-1163

[16]. Smith SC, Ladenson JH, Mason JW, Jaffe AS. Elevations of cardiac troponin I associated with myocarditis: experimental and clinical correlates. Circulation 1997;95:163-168

[17]. Lauer B, Niederau C, Kuhl U, et al. Cardiac troponin $\mathrm{T}$ in patients with clinically suspected myocarditis. J Am CollCardiol 1997;30:1354-1359 
[18]. Sheppard R, Bedi M, Kubota T, et al. Myocardial expression of fas and recovery of left ventricular function in patients with recentonset cardiomyopathy. J Am CollCardiol 2005;46:1036-1042

[19]. CrossRef $\mid$ Web of Science | Medline

[20]. Nishii M, Inomata T, Takehana H, et al. Serum levels of interleukin-10 on admission as a prognostic predictor of human fulminant myocarditis. J Am CollCardiol 2004;44:1292-1297

[21]. Morgera T, Di Lenarda A, Dreas L, et al. Electrocardiography of myocarditis revisited: clinical and prognostic significance of electrocardiographic changes. Am Heart J 1992;124:455-467

[22]. Nakashima H, Katayama T, Ishizaki M, Takeno M, Honda Y, Yano K. Q wave and non-Q wave myocarditis with special reference to clinical significance. Jpn Heart J 1998;39:763-774

[23]. Angelini A, Calzolari V, Calabrese F, et al. Myocarditis mimicking acute myocardial infarction: role of endomyocardial biopsy in the differential diagnosis. Heart 2000;84:245-250

[24]. Mendes LA, Picard MH, Dec GW, Hartz VL, Palacios IF, Davidoff R. Ventricular remodeling in active myocarditis: Myocarditis Treatment Trial. Am Heart J 1999;138:303-308

[25]. Felker GM, Boehmer JP, Hruban RH, et al. Echocardiographic findings in fulminant and acute myocarditis. J Am CollCardiol 2000;36:227-232

[26]. Mendes LA, Dec GW, Picard MH, Palacios IF, Newell J, Davidoff R. Right ventricular dysfunction: an independent predictor of adverse outcome in patients with myocarditis. Am Heart J 1994;128:301-307

[27]. Laissy JP, Messin B, Varenne O, et al. MRI of acute myocarditis: a comprehensive approach based on various imaging sequences. Chest 2002;122:1638-1648

[28]. Friedrich MG, Strohm O, Schulz-Menger J, Marciniak H, Luft FC, Dietz R. Contrast media-enhanced magnetic resonance imaging visualizes myocardial changes in the course of viral myocarditis. Circulation 1998;97:1802-1809

[29]. Cabinian AE, Kiel RJ, Smith F, Ho KL, Khatib R, Reyes MP. Modification of exercise-aggravated coxsackievirus B3 murine myocarditis by T lymphocyte suppression in an inbred model. J Lab Clin Med 1990;115:454-462

[30]. Maron BJ, Ackerman MJ, Nishimura RA, Pyeritz RE, Towbin JA, Udelson JE. Task Force 4: HCM and other cardiomyopathies, mitral valve prolapse, myocarditis, and Marfan syndrome. J Am CollCardiol 2005;45:1340-1345

[31]. Saegusa S, Fei Y, Takahashi T, et al. Oral administration of candesartan improves the survival of mice with viral myocarditis through modification of cardiac adiponectin expression. Cardiovasc Drugs Ther 2007;21:155-160

[32]. Tominaga M, Matsumori A, Okada I, Yamada T, Kawai C. Beta-blocker treatment of dilated cardiomyopathy: beneficial effect of carteolol in mice. Circulation 1991;83:2021-2028

[33]. Khatib R, Reyes MP, Smith F, Khatib G, Rezkalla S. Enhancement of coxsackievirus B4 virulence by indomethacin. J Lab Clin Med 1990;116:116-120

[34]. Costanzo-Nordin MR, Reap EA, O'Connell JB, Robinson JA, Scanlon PJ. A nonsteroid anti-inflammatory drug exacerbates Coxsackie B3 murine myocarditis. J Am CollCardiol 1985;6:1078-1082

[35]. Rezkalla S, Khatib R, Khatib G, et al. Effect of indomethacin in the late phase of coxsackievirus myocarditis in a murine model. J Lab Clin Med 1988;112:118-121

[36]. McNamara DM, Holubkov R, Starling RC, et al. Controlled trial of intravenous immune globulin in recent-onset dilated cardiomyopathy. Circulation 2001;103:2254-2259

[37]. Drucker NA, Colan SD, Lewis AB, et al. Gamma-globulin treatment of acute myocarditis in the pediatric population. Circulation 1994;89:252-257

[38]. Parrillo JE, Cunnion RE, Epstein SE, et al. A prospective, randomized, controlled trial of prednisone for dilated cardiomyopathy. N Engl J Med 1989;321:1061-1068

[39]. Stanton C, Mookadam F, Cha S, et al. Greater symptom duration predicts response to immunomodulatory therapy in dilated cardiomyopathy. Int J Cardiol 2008;128:38-41

[40]. Cooper LT Jr, Berry GJ, Shabetai R. Idiopathic giant-cell myocarditis -- natural history and treatment. N Engl J Med 1997;336:1860-1866

[41]. Wojnicz R, Nowalany-Kozielska E, Wojciechowska C, et al. Randomized, placebo-controlled study for immunosuppressive treatment of inflammatory dilated cardiomyopathy: two-year follow-up results. Circulation 2001;104:39-45

[42]. Oberg AL, Mahoney DW, Eckel-Passow JE, et al. Statistical analysis of relative labeled mass spectrometry data from complex samples using ANOVA. J Proteome Res 2008;7:225-233 